\title{
Fusion and reactions of exotic nuclei
}

\author{
${\text { I. } \text { Martel }^{1} \text {, E.F. Aguilera }}^{2}$, L. Acosta ${ }^{3}$, A.M. Sánchez-Benítez ${ }^{1}$ and R. Wolski ${ }^{4}$ \\ ${ }^{1}$ Departamento de Física Aplicada, Universidad de Huelva, E-21071 Huelva, Spain. \\ ${ }^{2}$ Departamento de Aceleradores, Instituto Nacional de Investigaciones Nucleares, 11801 México, D.F., México \\ ${ }^{3}$ INFN-Laboratori Nazionali del Sud, I-95123 Catania, Italy
}

\begin{abstract}
Close to the drip lines, the scattering cross sections of halo nuclei show a different behaviour as compared to the tightly bound projectiles of the stability line. Several experiments carried out in the last decade have been dedicated to investigate the competition between transfer, breakup and fusion channels at energies around and below the Coulomb barrier. The rather complex scenario gives rise to conflicting conclusions concerning the effect of breakup and transfer on reaction dynamics and the sub-barrier fusion process. In this work we discuss recent experimental findings in fusion and reactions of $6 \mathrm{He}$ halo nucleus at energies around the Coulomb barrier.
\end{abstract}

\section{Introduction}

The development of intense low energy radioactive beams has motivated an increasing interest in the study of reaction dynamics and the sub-barrier fusion of exotic nuclei $[1,2]$. Close to the drip lines, the loosely bound valence nucleons and their extended wave functions give rise to large radii exceeding by far the usual $A^{1 / 3}$ dependence. These features produce new phenomena, like exotic cluster structures, pigmy dipole resonances and the formation of skins and haloes. Therefore, preconceptions based on the highly successful experience of the reactions models on stable nuclei cannot be simply extrapolated to the scattering of exotic nuclei.

To achieve a better understanding of the reaction channels relevant for the dynamics of the fusion process of halo nuclei, high precision measurements must be performed in a systematic way. Among the various light halo nuclei candidates, the ${ }^{6} \mathrm{He}$ isotope has been one of the most widely studied due to its availability in a number of radioactive beam facilities: RIKEN (Japan), FLNR (Russia), REX-ISOLDE/CERN (Switzerland), CRC/LLN (Belgium), GANIL (France), TRIUMF (Canada), RIBRAS (Brasil).

Halo effects are absent in the collision between normal nuclei, and should clearly manifest when comparing the scattering observables of halo and nonhalo systems. For this purpose it is convenient to define specific scaling parameters that remove the effects due to different coulomb barriers and sizes of the systems being compared. In the following sections we review selected systematics of the fusion and reactions induced by the $2 n$ halo ${ }^{6} \mathrm{He}$ at near-barrier energies and relevant scaling methods.

\section{Angular distributions of elastic and alpha yields}

The combination of central and transition terms of the nuclear interaction between halo and heavy targets will produce strong static and dynamical effects at energies around the Coulomb barrier. A detailed study of the system ${ }^{6} \mathrm{He}+{ }^{208} \mathrm{~Pb}$ was performed recently at CRCLouvain-la-Neuve in different experiments ([3-6]). In these works the angular distribution of elastic and ${ }^{4} \mathrm{He}$ yields were measured in a range of energies around the Coulomb barrier $(14,16,18,22$ and $27 \mathrm{MeV})$. The angular distribution of the elastic cross sections relative to Rutherford at $22 \mathrm{MeV}$ is shown in Fig. 1 (data are from reference [5]). The elastic cross sections show a significant different behaviour as compared to the tightly bound stable ${ }^{4} \mathrm{He}$. A long-range absorption effect dumps the typical Fresnel diffraction pattern characteristic of heavy ion elastic cross sections. The data are properly described using a full CDCC calculation (pink solid line) and overestimated when dipole couplings are removed (blue solid line). The green line is a single channel calculation ignoring the couplings to the continuum. These results show that during the scattering process the electric field generated by a heavy target greatly distorts the wave function of the halo system, leading to dipole oscillations and strong effects due to the coupling to the continuum. 


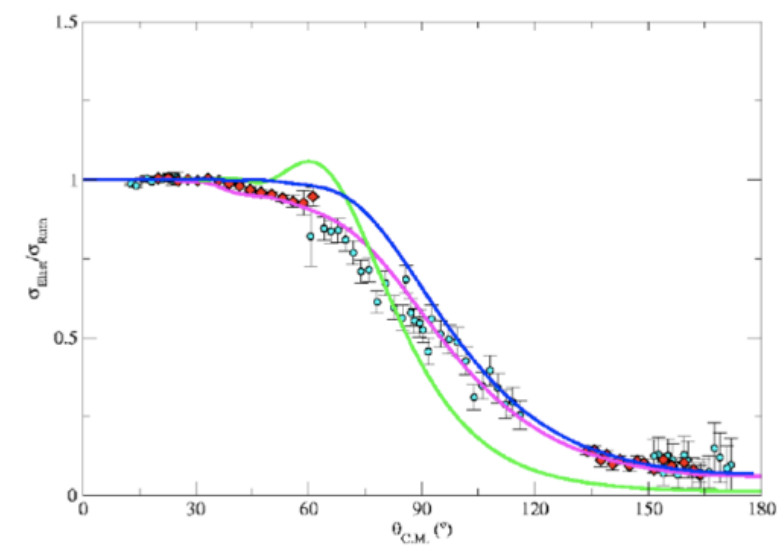

Fig. 1. Recent results of the scattering of ${ }^{6} \mathrm{He}+{ }^{208} \mathrm{~Pb}$ at $22 \mathrm{MeV}$ $[3,5]$. The angular distribution of elastic cross sections relative to Rutherford is presented. Solid lines: full CDCC calculation (pink), suppression of dipole coulomb coupling (blue), onechannel calculation (green). See text for discussion.

The angular distribution of ${ }^{4} \mathrm{He}$ yields relative to the elastic channel and the corresponding energy distribution is shown in Fig. 2. The data can be described (both the angular dependence and the energy distribution) in a DWBA approach by a two-neutron transfer process to the continuum states with $\mathrm{Q} \sim 0$ (solid line), assuming a dineutron model [4]. The results reveal the dominant role of this reaction channel. At sub-barrier energies the sequential two-neutron channel process will produce large effects [7].

The scattering system ${ }^{6} \mathrm{He}+{ }^{208} \mathrm{~Pb}$ also exhibits interesting regularities in the angular distributions of elastic and alpha production cross sections. For this purpose we show in Fig. 3 the available data for elastic scattering (circles) and alpha production (squares) cross sections (taken from [3-5]) relative to Rutherford. The angular distributions are presented as a function of the equivalent distance of closest approach $\mathrm{D}(\mathrm{fm})$ in a classical Coulomb trajectory. The distributions of both the elastic and ${ }^{4} \mathrm{He}$ yields follow a systematic trend despite being measured at different collision energies around the Coulomb barrier: 14, 16, 18, 22 and $27 \mathrm{MeV}$.

The $22 \mathrm{MeV}$ data were measured in the largest angular range, and therefore extends over the full radial range covered in the figure. At the other energies only limited angular regions at backward angles were covered. However, the figure shows that the data for these energies perfectly match the trend defined by the $22 \mathrm{MeV}$ data in their respective radial region. Within the experimental uncertainties, the sum (triangles) of the normalized elastic and alpha production cross section saturates to unity along the whole radial range. This is an indication that the contribution from other reaction channels, like the fusion channel, is small at these energies. It should be noticed how at $18 \mathrm{MeV}$ the elastic and breakup cross sections (green colour) reach the same value of 0.5 at angles around 180 degrees (D 13 fm).

For the case of $22 \mathrm{MeV}$, just above over the Coulomb barrier ( 20 MeV), two data sets are shown ([3] and [4]). The alpha yield measured in [4] is smaller than in [5] (although consistent within error bars) and the total cross section (elastic + alpha) relative to Rutherford becomes lower than one in this radial region. This result would indicate the presence of localized reaction channels not producing alpha particles at very backward angles (smaller distances of closer approach). A natural candidate would be the 2 n-evaporation fusion channel, as the fusion cross sections are high enough $(>\sim 100 \mathrm{mb})$ at energies above over the Coulomb barrier (Fig. 4) and the absorbed flux overcomes the experimental uncertainties.

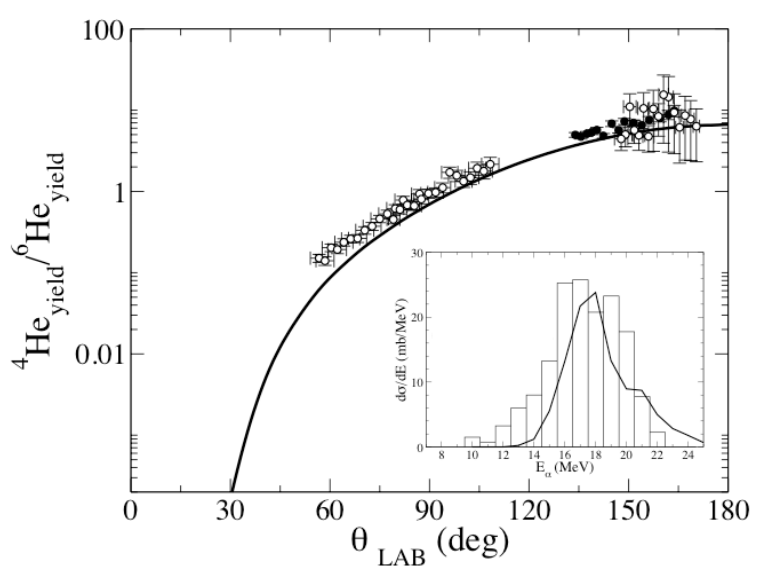

Fig. 2. Recent results of the scattering of ${ }^{6} \mathrm{He}+{ }^{208} \mathrm{~Pb}$ at $22 \mathrm{MeV}$ [4,5]. Angular distribution (Lab) of the relative ratio between yields of ${ }^{4} \mathrm{He}$ and ${ }^{6} \mathrm{He}$ (elastic). The solid line is a DWBA calculation for two-neutron transfer using the di-neutron model. The inset shows the energy distribution of ${ }^{4} \mathrm{He}$ fragments around 90 degrees (adapted from [5]). See text for discussion.

This systematic behaviour suggests the possibility to use simple phenomenological models to describe the scattering observables [8] with a suitable scaling technique $[19,20]$. In Fig. 3 we show with a solid line a parametric curve adjusted to describe the shape of the elastic cross sections. The dashed line, which follows the trend of the alpha production cross sections, was obtained by subtracting the former curve from unity. It is noticeable that a simple parametric shape can be used to estimate the angular distributions of both the elastic and alpha yields in such a wide range of energies around the Coulomb barrier. Our results could be understood by using a semi-classical picture, assuming a core-halo decoupling process. During the reaction process the ${ }^{6} \mathrm{He}+{ }^{208} \mathrm{~Pb}$ would follow Coulomb trajectories; at the classical turning point the neutron halo will not feel the strong Coulomb repulsion, thus leaving the ${ }^{4} \mathrm{He}$ core alone. At backward angles the neutrons would be transferred to continuum states of the system (by direct or sequential mechanism) with $\mathrm{Q} \sim 0$, the remaining alpha core following the corresponding coulomb trajectory. This process would be consistent with the result of DWBA calculations for neutron transfer [4,5] as well as with the measured energy distribution of ${ }^{4} \mathrm{He}$ fragments. The break process would dominate at forward angles.

Nevertheless the absorption produced by the fusion channel must be also reflected in the elastic and alpha yield distributions (as a loss of flux), but its relative contribution cannot be disentangled due to the actual experimental uncertainties. One should expect that by 
increasing statistics and reducing the error bars, it would be possible to discover the radial regions at which the fusion process is taking place. In this way a more complete picture of the relevant mechanisms of the scattering process could be obtained.

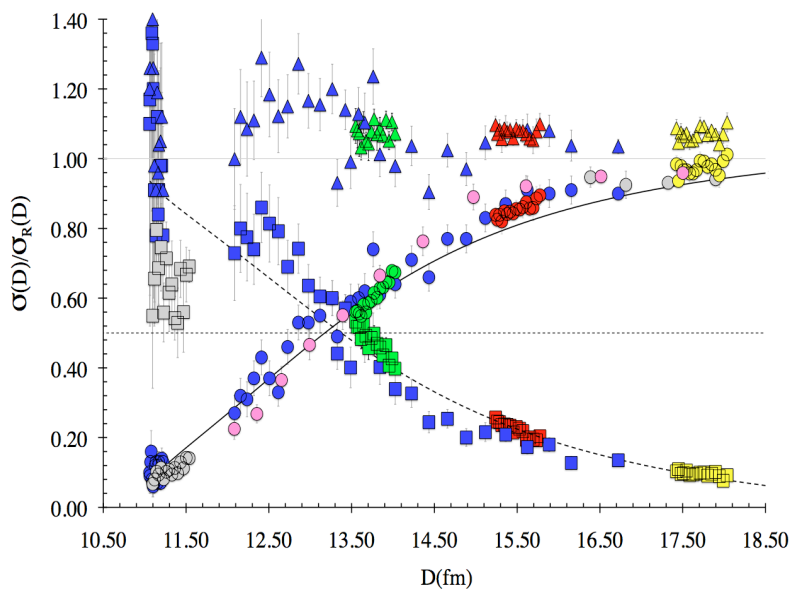

Fig. 3. Cross sections of elastic (circles) and ${ }^{4} \mathrm{He}$ yield (squares) for the ${ }^{6} \mathrm{He}+{ }^{208 \mathrm{P}} \mathrm{Pb}$ system, relative to Rutherford. Data are presented as a function of the distance of closest approach $\mathrm{D}(\mathrm{fm})$. The experimental results were obtained at several energies [3-6]: $14 \mathrm{MeV}$ (yellow), $16 \mathrm{MeV}$ (red), $18 \mathrm{MeV}$ (green) and $27 \mathrm{MeV}$ (pink). The data at $22 \mathrm{MeV}$ are shown with grey [4] and blue [5] colours. We also show the corresponding sum of the cross sections (triangles). The solid and dashed lines are parametric curves that follow the trend of the data. See text for discussion.

\section{Fusion and reaction cross sections}

Early attempts to describe the fusion phenomena where based on a tunneling process using potential barriers, often described by different approximations to the quantum mechanical penetration factors (see [1] for a recent review). In this way, a number of fusion recipes became popular and used broadly by the nuclear physics community. By selection of nuclear species with increasing number of neutrons one should expect a stronger nuclear interaction and an effective reduction of the Coulomb barrier, thus increasing the fusion probability. However, the addition of more neutrons does not necessarily lead to larger fusion cross sections, as demonstrated for the ${ }^{40,48} \mathrm{Ca}$ isotopes [9]. Coupling effects arising from specific details of the nuclear structure and dynamics of the systems involved can reduce the fusion yields for the richer neutron systems. An important reaction channel with strong effects on heavy ion fusion is the two-neutron sequential transfer process, and a successful description of the data can be only achieved by inclusion of this process in the reaction calculations [7].

In the case of the ${ }^{6} \mathrm{He}+{ }^{206} \mathrm{~Pb}$ system the predictions from the sequential two-neutron transfer model of V.I. Zagrebaev [7] lead to an extraordinary increase in the fusion channel at deep sub-barrier energies. At the experimental side several measurements of the fusion excitation function of ${ }^{6} \mathrm{He}$ with heavy targets $\left({ }^{206} \mathrm{~Pb},{ }^{209} \mathrm{Bi}\right.$, ${ }^{238} \mathrm{U}$ ) have been performed at different facilities. After the pioneering work of J.J. Kolata and E.F. Aguilera [10-12] using the TWINSOL facility at Notre Dame (USA), additional measurements were carried out by R. Raabe $[13,14]$ and R. Wolski [15] using the CRC facility at Louvain-la-Neuve (Belgium), and by Y.E. Penionzhkevich [16] and S.M. Lukyanov [17] using the radioactive beam facility at Flerov Laboratory of Nuclear Reactions (JINR, Dubna, Russia). The most recent results are shown in Fig. 4, where the two-neutron evaporation channel of the system ${ }^{6} \mathrm{He}+{ }^{208} \mathrm{~Pb}$ is compared with the corresponding ${ }^{4} \mathrm{He}+{ }^{208} \mathrm{~Pb}$ system (both producing the same compound nucleus ${ }^{212} \mathrm{~Pb}$ ). Despite the differences observed in the data sets, an overall enhancement of the fusion cross sections is observed when compared to the tightly bound ${ }^{4} \mathrm{He}$ isotope, in agreement with previous results [10-14].

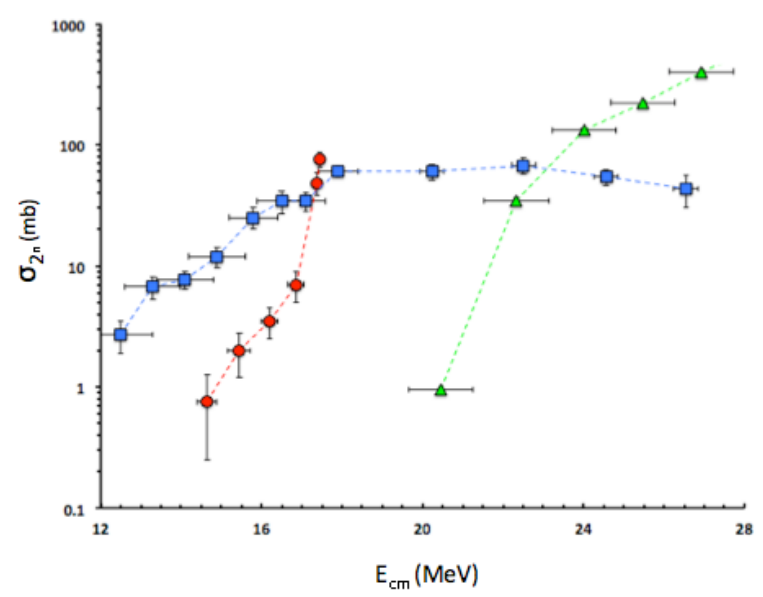

Fig. 4. Two-neutron evaporation cross sections for the ${ }^{6} \mathrm{He}+{ }^{206} \mathrm{~Pb}$ system: the red circles are the measurements of [15] (preliminary results), and the blue squares from [17]. The green triangles are the results of [17] for the tightly bound system ${ }^{4} \mathrm{He}+{ }^{208} \mathrm{~Pb}$. Lines with dashes have been drawn to guide the eyes. See text for discussion.

Fusion reactions from different systems can be properly compared using an energy scale defined by $\mathrm{X}$ $=\left(E-E_{b}\right) / \hbar \omega$, where $E_{b}$ is the barrier height at the barrier radius $R_{b}$, and $\hbar \omega$ the curvature. These parameters can be obtained from a suitable double folding model. The cross-section scaling factor is given by $\mathrm{Y}=2 \mathrm{E} /\left(\hbar \omega \mathrm{R}_{\mathrm{b}}{ }^{2}\right)$; with this formulation an universal fusion function (UFF) can be defined in terms of Wong's formula [18]. This parameterization has been successfully applied for a large number of systems, allowing for an elegant interpretation of the static and dynamical ingredients of the fusion process [19].

In the case of reaction cross sections a convenient prescription is given in [20]. The reaction cross sections are assumed to scale with projectile and target masses through a function of the form $\eta\left(A_{p}, A_{t}\right)=\alpha^{2}$, where $\alpha=$ $A_{p}{ }^{1 / 3}+A_{t}^{1 / 3}$. This quantity is proportional to the size of the colliding system and can be related to the equivalent hard sphere scattering cross section $\sigma_{\text {hd }}=\pi \eta$. For the collision energy the scaling factor is defined as $\xi\left(\mathrm{A}_{\mathrm{p}}, \mathrm{A}_{\mathrm{t}}, \mathrm{Z}_{\mathrm{p}} \mathrm{Z}_{\mathrm{t}}\right)=$ $\mathrm{Z}_{\mathrm{p}} \mathrm{Z}_{\mathrm{t}} / \alpha$, which is a measurement of the Coulomb barrier height $\mathrm{Vb}=1.3 \xi$. With this prescription it is found that the nuclear systems fall along one of three different 
groups: halo, weakly bound, and strongly bound nuclei. Each trajectory can be reproduced by a Wong's function fitted to the corresponding data.

An interesting concept arising form this scaling process is that of core-halo decoupling [21]: when the reaction takes place, the core and the halo interact independently with the target nucleus, so that the total reaction cross sections can be obtained by the addition of independent contributions arising from the core and the neutron halo. The halo contribution is given by the breakup and transfer yields of the corresponding reaction process. In order to evaluate the contribution of the core, the cross sections and energies of a given reaction involving the core nucleus on the same target is scaled using the reduced parameters defined above. Satisfactory results for the scattering of ${ }^{6} \mathrm{He}$ on heavy targets have been accomplished [22].

The appearance of this phenomenon is consistent with the dynamics of the collision process already discussed in Section 2: as the reaction process takes place, halo and core decouple and the cross sections add coherently along the corresponding trajectory.

\section{Summary and conclusions}

The angular distributions of elastic, breakup and transfer channels, together with the excitation functions of fusion and reaction cross sections reveal interesting features of the dynamics of halo nuclei at energies around the Coulomb barrier. The specific case of the ${ }^{6} \mathrm{He}$ system is particularly suitable for a systematic study using the data already available.

Data on elastic cross sections show a strong absorption pattern originated by coulomb dipole couplings and the effect of the coupling to the states of the continuum. Large alpha yields are obtained from neutron transfer mechanisms appearing mainly at backward angles. The fusion cross sections show a clear enhancement at deep sub-barrier energies with respect the tightly bound system ${ }^{4} \mathrm{He}+{ }^{208} \mathrm{~Pb}$.

The angular distributions of elastic and alpha yields, relative to Rutherford, show interesting regularities when are represented in terms of the distances of closest approach. This behaviour suggests the possibility of formulating universal functions for describing elastic and alpha yields at energies around the Coulomb barrier.

In the fusion process static and dynamical effects clearly appear when proper scaling is introduced. The reaction cross sections can be described by a parameterization based on Wong's formula by using suitable scaling parameters, and halo-core decoupling seems to be a useful concept for describing the reactions of halo systems.

As a summary, the availability of systematic sets of data on elastic, breakup, transfer and fusion cross sections of halo nuclei are essential to understand the dynamics of these complex systems. A number of new, high intensity radioactive beam facilities will be built in the near future: FRIB (USA), SPIRAL2 (France), FAIR (Germany), HIEISOLDE (CERN) and SPES (Italy). They should be able to provide the high quality data needed for one and two neutron halo systems at energies around the Coulomb barrier.

\section{References}

1. L.F. Canto et al., Phys. Rep. 424 (2006) 1.

2. N. Keeley et al., Prog. Part. Nucl. Phys. 63 (2009) 396.

3. A. Sánchez-Benítez et al., Nucl. Phys. A 803 (2008) 30 .

4. D. Escrig and et. al., Nucl. Phys. A 792, (2007) 2.

5. L. Acosta et al. Phys. Rev. C (2011), (submitted).

6. O. Kakuee and et. al., Nucl. Phys. A 339 (2003) 728.

7. V.I. Zagrebaev, Phys. Rev. C 67 (2003) 061601.

8. I. Martel et al., (in preparation).

9. M. Trotta et al., Phys. Rev. C 65 (2002) 011601.

10. J.J. Kolata et al., Phys. Rev. Lett. 81 (1998) 4580.

11. E.F. Aguilera et al., Phys. Rev. C 63 (2001) 061603(R).

12. E.F. Aguilera et al., Phys. Rev. Lett. 84 (2000) 5058.

13. R. Raabe et al., Nature 431 (2004) 823-826.

14. M. Trotta et al. Phys. Rev. Lett. 84 (2000) 2342.

15. R. Wolski et al., Eur. Jour. Phys. A, (submitted).

16. Y.E. Penionzhkevich et al., Phys. Rev. Lett. 96 (2006) 162701.

17. S.M. Lukyanov et al., Phys. Lett. B 670 (2009) 321.

18. L.F. Canto et al., J. Phys. G 36 (2009) 015109.

19. P.R.S. Gomes et al., Eur. Phys. Jour. (in this volume).

20. J. J. Kolata and E. F. Aguilera, Phys. Rev. C 79 (2009) 027603.

21. E.F. Aguilera et al., Phys. Rev. C 81 (2010) 011604(R).

22. E.F. Aguilera et al., Phys. Rev. C 83 (2011) 021601(R). 